\title{
Negotiation advantages of professional associations in health care*
}

\author{
Pedro Pita Barros \\ Universidade Nova de Lisboa \\ and CEPR (London)
}

\author{
Xavier Martinez-Giralt \\ Universitat Autònoma de Barcelona
}

July 2004

\begin{abstract}
In several instances, third-party payers negotiate prices of health care services with providers. We show that a third-party payer may prefer to deal with a professional association than with the sub-set constituted by the more efficient providers, and then apply the same price to all providers. The reason for this is the increase in the bargaining position of providers. The more efficient providers are also the ones with higher profits in the event of negotiation failure. This allows them to extract a higher surplus from the third-party payer.
\end{abstract}

JEL numbers: I11, I18

Keywords: professional associations, negotiation, health care.

CORRESPONDENCE ADDRESSES:

Pedro Pita Barros

Xavier Martinez-Giralt

Departamento de Economia CODE and Departament d'Economia

Universidade Nova de Lisboa Universitat Autònoma de Barcelona

Travessa Estêvão Pinto

Edifici B

P-1099-032 Lisboa

08193 Bellaterra

Portugal

Spain

Fax: 351-21-388 6073

Fax: 34-93-581 2012

Email: ppbarros@fe.unl.pt

xavier.martinez.giralt@uab.es

\footnotetext{
${ }^{*}$ We benefited from the comments and suggestions of Steffen Hoernig, Pau Olivella, Vasco Santos, two anonymous referees, and seminar participants. The financial support of Fundación BBVA, and projects SAPIENS grant 37762/01 (Pedro P. Barros) and 2001SGR-00162, and BEC2003-01132 (Xavier Martinez-Giralt) is gratefully acknowledged. The usual disclaimer applies.
} 


\section{Introduction.}

The simultaneous existence of a public financing entity (third-party payer) and private providers of health care motivates the existence of contracts governing the relationship between the third-party payer and the providers.

A popular contractual form is the setting of a fee for service - the financing institution pays a pre-determined amount for a given service per patient treated. Despite a general trend towards different contractual forms, in some countries, and for certain services provided, this approach is still dominant. ${ }^{1}$

In public systems, it is often the case that the National Health Service (NHS) contracts with private providers the provision of health care services. Typically, the value of the fee is set in a negotiation procedure between the NHS and either an association representing providers or some providers individually. An interesting economic question here is whether the NHS would do better negotiating with an association instead of selecting the largest companies as preferential partners. At first sight, negotiating with the largest companies, which are also more efficient in production, may lead to lower prices. These firms can accommodate lower prices due to lower production costs. Negotiating with an association would mean that the interests of smaller, inefficient, companies would be considered, driving prices up. It is important to keep in mind that professional associations may act as devices to disclose information (mostly aggregate) for its members, but not as collusive devices. It is beyond the scope of our analysis to go into the governance rules of professional associations.

This view, however, ignores the fact that the more efficient companies may be tougher negotiators, and thus obtain a better (higher) price, which is extended afterwards to all other companies. This is the case, for instance, in the Portuguese dialysis sector where the NHS negotiates the price of a dialysis session with the two largest companies and extends the agreed price to all companies. The bargaining strength comes from the fallback value (outside option) in case of failure

\footnotetext{
${ }^{1}$ See Mossialos and Le Grand (1999, pp.17-19) for a review of payment systems for health care providers in the European Union.
} 
in negotiations. For instance, assuming that patients will be treated, even if at the cost of direct payments, the more efficient companies will have relatively higher profits. Thus, they will be more demanding in negotiations than a sectoral association, because the latter takes into account the relatively low profits of the less efficient companies. Consequently, the association is willing to concede a less favorable surplus division in order to avoid failure of negotiations. In other words, the negotiation with the more efficient firms may benefit all providers and lead to higher expenditure by the NHS. If, alternatively, we assume that patients will not be treated by these providers in the case of negotiation failure, then the reinforcement of bargaining power of providers associated with the negotiation procedure including only the more efficient ones does not exist. ${ }^{2}$ There remains only the first effect: more efficient firms are more willing to take lower prices. In this case, the NHS benefits from negotiating with the more efficient providers only, instead negotiating with a sectoral association.

A seemingly attractive alternative system is to reimburse patients. However, doing so leads to higher prices than under the negotiation process (as companies have pricing freedom and patients, under full insurance, are insensitive to price differences). ${ }^{3}$ In our motivating example, the nature of the disease justifies the presumption that patients will be treated even if by own-pocket payment (chronic renal insufficiency, if not compensated by dialysis or a kidney transplant, leads to death). Thus, we conclude that the NHS should negotiate with a sectoral association and not with the more efficient, largest, providers. As it will be apparent from the formal analysis, the main insight is not specific to this example, being applicable whenever a third-party payer negotiates with health care providers.

We still have an open question to tackle. This refers to the reason why the thirdparty payer may want to participate in a negotiation. In our model individuals have an ex-ante probability of getting sick. Once these (individual) probabilities are realized we have a population of patients seeking treatment. We concentrate in this part of the game rather than in the full game. As usual in this situations, issues of

\footnotetext{
${ }^{2}$ The NHS may resort to its own facilities, for example.

${ }^{3}$ See Barros and Martinez-Giralt (2002).
} 
universal access to health care services, equity, adverse selection, etc. arise. Thus, there appears a demand for insurance providing a role for third-party payers. That role creates economic value, which is shared with providers by means of higher prices.

There are, of course, other mechanisms to determine the price from the thirdparty payer's point of view. Alternatives like "any willing provider" type of contracts are explored in a companion paper, Barros and Martinez-Giralt (2000). The aim of this paper though is to provide some rationale for the particular way to determine prices in the situation described.

The next sections are devoted to the formal presentation of the argument. The second section shows that without asymmetries, it is irrelevant whether the negotiation is done with a subset of companies or with an association. The third section introduces cost asymmetries and establishes our main result. Next, the fourth section shows the result to be reversed if patients are not treated in the event of negotiation failure. Section four deals explicitly with the issue of the investment in capacity by the public sector. Finally, section six presents some concluding remarks.

\section{The model.}

We consider a setting where a third-party payer, say a National Health Service (NHS), has to negotiate prices of health care services with providers. We assume, for the moment, zero production costs in the provision of health care and the existence of two providers. The former assumption is relaxed in section 3.

Price negotiation can be carried out under two different regimes. In the first one, the providers join a sectoral or professional association. The association negotiates the price with the NHS. In the second regime, the NHS negotiates the price with one provider and extends the agreed price to the contract involving the other provider. In particular, when cost asymmetries exist we assume the NHS to negotiate with the more efficient provider. This assumption and its implications are discussed in the final section. The negotiation outcome is described by the Nash 
bargaining solution. ${ }^{4}$

In the case of failure to reach an agreement, both providers compete in the market. Competition takes place on price. Since we consider cases where demand is essentially exogeneous and has to be fully satisfied, this is a more natural assumption than quantity competition. ${ }^{5}$ We assume providers to be characterized by horizontal product differentiation as perceived by the consumer. Differentiation can be due to geographical distance and/or subjective preferences of the consumer, for example. This means that we model market interaction as a Hotelling product differentiation situation. Providers are located at the endpoints of a segment $[0,1]$. Patients are uniformly distributed along the line, with unit mass.

Consumers are insured and face a copayment rate $s$. This assumption is innocuous for the analysis. Actually, all the qualitative results hold for any value $s \in(0,1)$. Note that $s=1$ implies no insurance to patients, which would be contradictory with the role of the third-party payer.

The NHS has a budget $M$ from which it must pay providers. Having free funds is positively valued by the NHS as it allows for its productive application elsewhere in the health sector. The gain to the NHS from the negotiation is given by the difference in the net surplus under negotiation and in the case of failure. We denote by $R$ such value net of the fallback value. In our simple model, given the assumption that a positive level of insurance coverage is always guaranteed to patients, it will be the payment to be made by the NHS to ensure provision in the private market plus the value, in monetary terms, of the extra insurance level provided to patients (a copayment $s$ ) ${ }^{6}$

We assume profits of both providers to be equally weighted in the objective function of the association. An alternative assumption would be to assume that the more efficient provider has a larger influence in the association's objectives. This would leave the qualitative results unchanged, as it would fall between our two

\footnotetext{
${ }^{4}$ Extensive presentations of the non-cooperative bargaining theory can be found in Binmore et al. (1986), Osborne and Rubinstein (1990) and Roth (1985), among others.

${ }^{5} \mathrm{We}$ assume that even in the presence of an association, providers do not collude. We will discuss this assumption later.

${ }^{6}$ See section on insurance value in the Appendix for a formal derivation.
} 
polar cases.

Denote by $\Pi_{i}, i=A, B$ the profits of each provider and by $\bar{\Pi}_{i} ; i=A, B$ their profits in the case of negotiation failure. Profits are then given by

$$
\Pi_{A}=x p_{A}, \quad \Pi_{B}=(1-x) p_{B},
$$

where $p_{i}, i=A, B$, is the price received by each provider and $x$ is the patient indifferent between providers $A$ and $B$. This indifferent patient is defined by,

$$
x=\frac{1}{2}-\frac{s\left(p_{A}-p_{B}\right)}{2 t} .
$$

Parameter $t$ reflects product differentiation, and it is modeled as the (linear) "transport cost" of not consulting the most preferred type of provider. ${ }^{7}$

According to our assumptions, when the NHS negotiates with an association that takes into account the interests of both players, the equilibrium price, $p$, is the solution to the following program:

$$
\max _{p} \Omega=\left(R-\Pi_{A}-\Pi_{B}\right)^{\delta}\left(p-\bar{\Pi}_{A}-\bar{\Pi}_{B}\right)^{1-\delta}=(R-p)^{\delta}(p-t / s)^{1-\delta},
$$

where $R \equiv \frac{(1-s) t}{s}+I$, and $\delta \in(0,1)$ denotes the bargaining power of the NHS. The first part in $R$ denotes the payment to private providers in the private market equilibrium and $I$ the insurance value to patients. The second equality follows from $\Pi_{A}+\Pi_{B}=p$, and $\bar{\Pi}_{A}+\bar{\Pi}_{B}=t / s$. The first expression comes from the fact that all patients are treated at price $p$ and treatment costs are zero by assumption. The second one results from the private market equilibrium, which yields an equilibrium profit of $t / 2 s$ for each provider.

Note that the price charged by the providers is higher the higher differentiation is and the more insulated consumers are from the price (lower $s$ ). This is the reason why $t$ and $s$ show up in $R$ - the insurance premium must cover the price charged by the provider. ${ }^{8}$ Also, since costs are normalized to zero, the payments made by the NHS equal the revenues of the firms, which are equal to the profits.

\footnotetext{
${ }^{7}$ See Hotelling (1929) or a textbook treatment such as Tirole (1988).

${ }^{8}$ The equilibrium price is set at $t / s$, as we assumed, for simplicity and without loss of generality, zero marginal cost of treatment and the NHS pays only a fraction $(1-s)$ of the price. See section on insurance value in the Appendix.
} 
The first-order condition of the above program can be written as: ${ }^{9}$

$$
\frac{\partial \Omega}{\partial p}=-\delta(R-p)^{\delta-1}\left(p-\frac{t}{s}\right)^{1-\delta}+(1-\delta)(R-p)^{\delta}\left(p-\frac{t}{s}\right)^{-\delta}=0 .
$$

which, after simplifications yields:

$$
-\delta(p-t / s)+(1-\delta)(R-p)=0 .
$$

Thus, the equilibrium price is:

$$
p^{*}=\delta t / s+(1-\delta) R \text {. }
$$

Consider now the case where the NHS negotiates with, say, provider $A$ and applies the resulting price to provider $B$, as well. The program to be solved is:

$$
\begin{aligned}
\max _{p} \Omega= & \left(R-\Pi_{A}-\Pi_{B}\right)^{\delta}\left(\Pi_{A}-\bar{\Pi}_{A}\right)^{1-\delta}=(R-p)^{\delta}(p / 2-t / 2 s)^{1-\delta} \\
& \text { s.t. } \quad \Pi_{B} \geq 0 .
\end{aligned}
$$

The second equality follows from $\bar{\Pi}_{A}=1 / 2 t / s, \Pi_{A}+\Pi_{B}=p$, and equal split of demand between providers when equal prices are set, meaning that $\Pi_{A}=p / 2$.

Note that although the NHS negotiates only with provider A, it takes into account that the same price will apply to the other provider. A further requirement is that provider $B$ makes non-negative profits. Thus, market demand will be equally split. The expression above makes use of profit definitions. It is clear that it yields the same solution as the first program. Since firms are symmetric, any price that gives non-negative profits to provider $A$ also ensures the constraint to be satisfied. The negotiation will, in fact, give strictly positive profits to both providers. Thus, under symmetry of providers, the NHS and providers are indifferent between the two alternative procedures. The next section departs from this symmetric world.

\footnotetext{
${ }^{9}$ It is straightforward to check that the second-order condition for a maximum of this program holds.
} 


\section{Negotiating with asymmetric providers.}

We now assume that provider $A$ is more cost efficient. Provider $B$ has a production $\operatorname{cost} c>0$ per patient treated whereas provider $A$ remains with zero cost. Now, the equilibrium in a private market without a third-party payer is not symmetric. It is characterized by ${ }^{10}$

$$
p_{A}=\frac{c s+3 t}{3 s}, p_{B}=\frac{3 t+2 c s}{3 s} \text { and } \bar{\Pi}_{A}=\frac{(c s+3 t)^{2}}{18 t s}, \bar{\Pi}_{B}=\frac{(3 t-c s)^{2}}{18 t s} .
$$

The above profits define the fallback values for the providers in case of negotiation failure. It may be the case that the NHS does not have a precise knowledge on firms marginal costs. Nevertheless, appealing to data like market shares, the facilities provided by the different providers, the cash-flow, etc., the NHS may infer the relative position of providers in terms of efficiency. We stylize this situation by assuming that the firms' costs are known by the NHS.

When the NHS negotiates with the sectoral/professional association, the equilibrium solves the following program:

$$
\max _{p} \Omega=(R-p)^{\delta}\left(p-\frac{c}{2}-\frac{(c s+3 t)^{2}}{18 t s}-\frac{(3 t-c s)^{2}}{18 t s}\right)^{1-\delta},
$$

where now $R \equiv(1-s)\left(x p_{A}+(1-x) p_{B}\right)+I$, and $x$ is the indifferent consumer between providers $A$ and $B$. It is given by $x=\frac{s\left(p_{B}-p_{A}\right)}{2 t}+\frac{1}{2}$, and $p_{A}$ and $p_{B}$ are the private market prices of providers $A$ and $B$ respectively. Solving the first-order condition yields the equilibrium price:

$$
p^{+}=(1-\delta) R+\delta\left(\frac{c}{2}+\frac{(c s+3 t)^{2}}{18 t s}+\frac{(3 t-c s)^{2}}{18 t s}\right) .
$$

Equilibrium profits are given by,

$$
\Pi_{A}=p^{+} / 2 ; \Pi_{B}=\left(p^{+}-c\right) / 2 .
$$

Consider now the negotiation with the more efficient provider, which is also the largest one. The price determined by the negotiation applies to both providers.

\footnotetext{
${ }^{10}$ See section on private market equilibrium with asymmetric providers in the appendix.
} 
We assume the third-party payer wants to have all providers active. The program to be solved is:

$$
\begin{gathered}
\max _{p} \Omega^{\prime}=(R-p)^{\delta}\left(\frac{p}{2}-\frac{(3 t+c s)^{2}}{18 t s}\right)^{1-\delta} \\
\text { s.t. } \quad \frac{1}{2}(p-c) \geq 0 .
\end{gathered}
$$

Let us first consider the problem without the constraint of non-negative profits for provider $B$. Following this, we will show that provider $B$ has strictly positive profits as well.

The first-order condition leads, after manipulation, to the following equilibrium price:

$$
p^{\prime}=(1-\delta) R+\delta \frac{(c s+3 t)^{2}}{9 t s} .
$$

We know from the bargaining process that the equilibrium price must be such that

$$
p>\frac{(3 t+c s)^{2}}{9 t s} .
$$

Thus, if

$$
\frac{(3 t+c s)^{2}}{9 t s}-c>0
$$

holds, then provider $B$ makes strictly positive profits. This condition can be rewritten as:

$$
c^{2} s^{2}+3 t(3 t-c s)>0 \text {. }
$$

In the private-market equilibrium, prices must cover costs. Thus, from (1), we require $3 t-c s>0 .{ }^{11}$ Hence, condition (3) always holds and the non-negative profit constraint in problem (2) is not binding in equilibrium.

It is straightforward to see that,

$$
p^{\prime}-p^{+}=c \delta / 6>0 \text {. }
$$

Thus, the price is lower when the NHS negotiates with an association. Since a uniform price is set in both cases, demand is evenly split between the two providers, and both earn higher profits if the NHS negotiates with the more efficient one.

\footnotetext{
${ }^{11}$ Even if this condition does not hold, the qualitative features of the equilibrium are the same. See section on equilibrium characterization in the Appendix for a proof of this claim.
} 
The result hinges on the tougher position taken by the more efficient provider. Since it is relatively more efficient it has less to lose in the event of negotiation failure. This drives the price up, and more than compensates the downward effect of lower costs of production.

\section{Providers without outside option.}

To see that the main effect comes through the bargaining position induced by a better fallback value, consider the following alternative situation in case of negotiation failure: the NHS is able to totally divert patients to other treatment alternatives. ${ }^{12}$ In terms of the negotiation process, this means that negotiation failure leads to zero demand for both providers. In this case, the fallback value for both providers is zero. Let us now denote by $\mu$ the cost of treatment at the public service. Computations similar to those in the previous section show the equilibrium price to be, when the NHS negotiates with an association,

$$
p^{+}=\mu(1-\delta)+\delta c / 2,
$$

while the equilibrium price if the NHS negotiates with the more efficient provider is:

$$
p^{\prime}=\mu(1-\delta) .
$$

It follows directly that

$$
p^{\prime}<p^{+} .
$$

From the point of view of the third-party payer, it is better to negotiate with the more efficient provider and apply that price to the second provider. The result reverses that of the previous section. The crucial difference is that, in the latter situation, shifting from negotiating with an association to negotiating with the more efficient provider does not change the fallback value of providers in case of a breakdown in negotiations. The mechanism that weakened the position of the NHS does not exist here.

\footnotetext{
${ }^{12}$ This may imply a higher cost. If so, $R$ can also differ from the previous case. In particular, it would be higher in the current section than in the previous one. Such a case would strengthen the case made in Section 3. The price comparison would not be clear-cut.
} 


\section{Concluding remarks.}

We addressed a simple, yet economically significant, question: should a NHS (or a third-party payer, in general) negotiate prices of health care services with professional associations, or should it negotiate only with the more efficient ones and apply the resulting price to all providers? The first alternative has been common, but the second one can also be found in the health care sector.

We showed that the apparent benefit of negotiating with the more efficient providers (obtaining lower prices) can be more than outweighed by a stronger bargaining position of the provider, when compared to dealing with an association. This is so because a representative association also incorporates in its decisions the (relatively larger) decline in profits of the less efficient firms in the event of negotiation failure.

The policy implication is that the NHS should avoid negotiating with the largest providers, if they are significantly more efficient and have a valuable outside option in the private market (a possibility in case of chronic conditions and lack of capacity in the public system). Instead, it should promote negotiations over prices with an association representative of all providers' interests. According to our findings, all providers benefit from partial negotiation with the efficient firms. So, the association will not take over price negotiations without pressure from the NHS for that to happen.

From the point of view of the providers, they should try to force a negotiation of the NHS with the largest ones. In the Portuguese dialysis sector, the NHS negotiates prices for each dialysis session with the two largest providers. The price settled in this agreement is then applied to all companies. Surprisingly enough, the smaller companies have not been claiming a role in the price-determination process. Given that it is reasonable to assume the largest providers are the more efficient ones, being subsidiaries of vertically integrated multinationals, our model presents an explanation for the current satisfaction of all firms with the status quo. All firms benefit from the tougher position of the largest firms, compared to what would be the stance of an association including all providers. Thus, it seems that 
these providers are able to force the terms of the negotiation on the NHS.

Naturally, the existence of negotiations between the payer and professional associations in health care is not specific to Portugal or to dyalisis. We found it in Belgium for determination of hospital fees, of specialized ambulatory care and dental care, for example (Crainich and Closon, 1999). Also in Germany, France and Austria, negotiations took place between payers and representatives of providers of ambulatory care. ${ }^{13}$.

Some caveats apply. Given our results, it is tempting to draw another policy implication. By reversing the argument, the NHS should attempt to negotiate with the less efficient providers and then apply this price to all providers. In this case, we can show that it is also better to negotiate with the professional association. Nevertheless, this alternative seems to be quite difficult to implement, especially if the less efficient providers are also the smaller ones in the market. In addition, if the inefficiency is large enough, a excessively high price may result anyway. Thus, considering that the NHS has the option, negotiating with the more efficient/largest providers seems the more reasonable choice.

A second issue that was not treated explicitly is the governance and decisionmaking mechanisms of the association, as well as the membership decision by providers. This is left for future research, though we conjecture the main forces identified here will not be reversed.

A third aspect to be discussed is the assumption that providers do not collude in the market, even in the presence of an association. This is reasonable as competition rules explicitly forbid such role for sectoral associations. Nonetheless, if we allow for collusion, in the case of negotiation failure, prices will be equal to the reservation price of patients (or an even higher amount if there is a reimbursement rule by the third-payer). Since out-of-pocket payments put patients at financial risk, there is room to negotiate prices above this collusive level. The major difference to our analysis is that, under collusion, breakdown of negotiations does not introduce

\footnotetext{
${ }^{13}$ In Germany, the negotiation takes place between sickness funds and physicians' associations (Busse and Howorth, 1999); in France, there were the conventions between Assurance-Maladie and private doctors unions (Lancry and Sandier, 1999); and in Austria, between the regional chambers of doctors and the social insurance funds (Engelbert, 1999)
} 
product market asymmetries across providers. The third-party payer would be indifferent between negotiating with an association or with a sub-set of providers, extending afterwards the settled price to all providers. ${ }^{14}$

Another assumption deserving discussion is that only one negotiation is carried out. This implies that there is no room for price discrimination. Alternatively, one could think of a sequential bargaining procedure. In such a case, the NHS would first negotiate with one provider and then with the other. We provide some intuition for two alternative scenarios. Negotiate with the most efficient provider is better when after failing in the bargaining with the more efficient provider, the third-party payer negotiates with the less efficient provider and excludes the former from coverage. Also, if the negotiated price with the less efficient provider is extended to the more efficient provider, then again it is more advantageous for the third-party payer to negotiate with the more efficient provider only rather than engage in sequential negotiations. In the analysis we also rule these situations out because price discrimination on the fee per session is typically seen as undesirable and usually faces strong opposition by providers. Also, conducting sequential negotiations adds considerably to transaction costs. The settlement of prices may take several months and involves the use of real resources by both parties. Taking these two elements together, we find it reasonable to assume that only one negotiation takes place and the resulting price applies to all providers.

Summarizing, whenever a third-party payer negotiates prices with providers, it will do better by dealing with an association inclusive of all providers if the providers are asymmetric in production costs and face valuable outside options, in comparison with negotiating with a set of the more efficient providers. This finding reassesses the role of professional associations in price determination processes, at least in some health care markets. It does not mean that our insight applies to every price negotiation that exists in the health care sector. It does call our attention to some subtleties that have been so far ignored.

\footnotetext{
${ }^{14}$ If some providers can be excluded, we fall in the analysis of Barros and Martinez-Giralt (2000).
} 


\section{References}

Barros, P.P. and X. Martinez-Giralt, 2002, Public and private health care providers, Journal of Economics and Management Strategy, 14(1): 109 - 133.

Barros, P.P. and X. Martinez-Giralt, 2000, Selecting negotiation processes with health care providers, manuscript, available at $\langle$ http://ppbarros.fe.unl.pt/investigacao/research.html $\rangle$.

Binmore, K.G., A. Rubinstein and A. Wolinsky, 1986, Non-cooperative models of bargaining, in Handbook of Game Theory with Economic Applications, edited by R.J. Aumann and S. Hart, Amsterdam, North-Holland.

Busse, R. and C. Howorth, 1999, Cost containment in Germany: twenty years experience, in Health care and cost containment in the European Union edited by E. Mossialos and J. Le Grand, Ashgate Publishing Limited, pp. $303-339$.

Crainich, D. and M-C. Closon, 1999, Cost containment and health care reform, in Health care and cost containment in the European Union edited by $\mathrm{E}$. Mossialos and J. Le Grand, Ashgate Publishing Limited, pp. 219 - 266.

Dixit, A., 1979, A model of duopoly suggesting a theory of entry barriers, Bell Journal of Economics, 10: 10 - 20.

Dixit, A., 1980, The role of investment in entry deterrence, Economic Journal, 90: $95-106$.

Engelbert, T., 1999, Expenditure and cost control in Austria, in Health care and cost containment in the European Union edited by E. Mossialos and J. Le Grand, Ashgate Publishing Limited, pp. 605 - 633.

European Observatory on Health Care Systems, 1999, Health Care Systems in Transition - Portugal.

Folland, S., A.C. Goodman and M. Stano, 2001, The Economics of Health and Health Care, 3rd Edition, Prentice Hall

Hotelling, H., 1929, Stability in Competition, Economic Journal, 39: 41 - 57.

Lancry, P.-J. and S. Sandier, 1999, Twenty years of cures for the French health care system, in Health care and cost containment in the European Union edited by E. Mossialos and J. Le Grand, Ashgate Publishing Limited, pp. 443 478.

Mossialos, E. and J. Le Grand, 1999, Cost containment in the EU: an overview, in Health care and cost containment in the European Union edited by E. Mossialos and J. Le Grand, Ashgate Publishing Limited, chapter 1.

Osborne, M.J. and A. Rubinstein, 1990, Bargaining and markets, San Diego, Academic Press.

Roth, A.E., 1985, Game-theoretic models of bargaining, Cambridge (Mass.), Cambridge University Press. 
Spence, A.M., 1977, Entry, capacity, Investment, and oligopolistic pricing, Bell Journal of Economics, 8: 534 - 544.

Spence, A.M., 1979, Investment strategy and growth in a new market, Bell Journal of Economics, 10: 1 - 19.

Tirole, J., 1988, The Theory of Industrial Organization, Cambridge (Mass.), The MIT Press. 


\section{Appendix.}

\section{Insurance value}

We consider a situation where a NHS is financed by contributions from the population, either through the tax system or earmarked contributions. Utility of consumers is given by an utility function $U($.$) with positive marginal utility and risk$ aversion. Let $Y$ be income and $T(Y)$ be the financial contribution to the health system.

In the case of negotiation failure, and insurance coverage with copayment $s$, the expected utility is

$$
E U_{1}=q U(Y-T(Y)-t)+(1-q) U(Y-T(Y)),
$$

where $q$ is the probability of being sick and $t / s$ the private market equilibrium price if patients have to pay a fraction $s$ of the price $(s \times t / s$ is then total copayment by patients). If negotiations succeed, the patient receives full insurance and has expected utility given by $E U_{2}=U(Y-T(Y))$

The objective function of the National Health Service is given

$$
W=M-p+\lambda E U
$$

where $E U$ denotes expected utility of patients, $M$ the financial value available, and $\lambda \in(0,1)$ reflect the weight of consumers' utility on the objective function of the NHS. the financial value $M$ can be seen as resulting from the patients' contributions $M=\int_{Y} h(Y) T(Y) d Y$ where $h$ is the density function of income distribution in the population. Both $p$ and $E U$ determine the fallback value for the NHS, $\bar{W}:$

$$
\bar{W}=M-(1-s) \frac{t}{s}+\lambda E U_{1}
$$

Then,

$$
\begin{array}{r}
W-\bar{W}=\lambda q[U(Y-T(Y))-U(Y-T(Y)-t)]+(1-s) \frac{t}{s}-p= \\
I+(1-s) \frac{t}{s}-p,
\end{array}
$$


where $I$ represents the insurance value to patients. Finally, we define

$$
R \equiv I+(1-s) \frac{t}{s}
$$

\section{Private market equilibrium with asymmetric providers}

Assume provider $A$ is more efficient than provider $B$ who faces a production cost $c>0$. The indifferent patient, as usual, is given by

$$
x\left(p_{A}, p_{B}\right)=\frac{s\left(p_{B}-p_{A}\right)}{2 t}+\frac{1}{2} .
$$

Profits are now defined by,

$$
\bar{\Pi}_{A}=p_{A} x\left(p_{A}, p_{B}\right), \text { and } \bar{\Pi}_{B}=\left(p_{B}-c\right)\left(1-x\left(p_{A}, p_{B}\right)\right) .
$$

First order conditions yield,

$$
\begin{aligned}
& p_{A}\left(p_{B}\right)=\frac{t}{2 s}+\frac{p_{B}}{2} \\
& p_{B}\left(p_{A}\right)=\frac{t+c s}{2 s}+\frac{p_{A}}{2} .
\end{aligned}
$$

Solving for prices we obtain,

$$
p_{A}=\frac{3 t+c s}{3 s}, \text { and } p_{B}=\frac{3 t+2 c s}{3 s} .
$$

Substituting those prices in the expression of the indifferent patient we obtain the equilibrium profits,

$$
\bar{\Pi}_{A}=\frac{(3 t+c s)^{2}}{18 t s} \text {, and } \bar{\Pi}_{B}=\frac{(3 t-c s)^{2}}{18 t s} .
$$

\section{Equilibrium characterization}

Suppose that $3 t-c s<0$ in Section 3. This means that $p_{B}<c$ in the private market. That is, if negotiations between providers and the third-party payer break down, the private market outcome results in the least-efficient provider being driven out of the market. 
Assuming that the reservation price is high enough, the remaining provider serves the entire market at price $p=c-t / s$. Even the most distant patient prefers the more efficient provider. ${ }^{15}$ Profits of the more efficient provider are, in this case, $\Pi=c-t / s$, as it gets all demand.

This price is higher than the one under duopoly for the more efficient provider $(c-t / s>(3 t+c s) / 3 s$ under $c s>3 t)$. Then, we have $\bar{\Pi}_{A}=c-t / s$. The bargaining solution, when the third-party payer negotiates only with the more efficient provider solves:

$$
\begin{aligned}
& \max _{p} \Omega=(R-p)^{\delta}\left(\frac{1}{2} p-c+t / s\right)^{1-\delta} \\
& \text { s.t. } \quad p \geq c .
\end{aligned}
$$

The constraint ensures that the second provider is active in the market. Let us ignore for the moment the constraint. The associated first-order condition is,

$$
-\delta\left(\frac{1}{2} p-c+t / s\right)+(1-\delta) \frac{1}{2}(R-p)=0 .
$$

The equilibrium price is, therefore,

$$
p^{*}=(1-\delta) R+2 \delta(c-t / s) .
$$

For this to be a well-defined equilibrium, one has to have $R-p^{*}>0$, which originates the condition $R>2(c-t / s)$. Computation of $p^{*}-c$ yields,

$p^{*}-c=(1-\delta) R+2 \delta(c-t / s)-c>2(1-\delta)(c-t / s)+2 \delta(c-t / s)-c=(c s-2 t) / s>0$,

where the inequalities follow from the above conditions.

Consider now the negotiation with an association:

$$
\begin{aligned}
\max _{p} \Omega= & (R-p)^{\delta}\left(p-\frac{1}{2} c-c+t / s\right)^{1-\delta} \\
& \text { s.t. } \quad p \geq c .
\end{aligned}
$$

The first-order condition is,

$$
-\delta\left(p-\frac{3}{2} c+t / s\right)+(1-\delta)(R-p)=0 .
$$

\footnotetext{
${ }^{15}$ To be rigorous, $p=c-t / s-\varepsilon$, where $\varepsilon$ is the smallest unit available to denominate prices.
} 
From it, we obtain the equilibrium price:

$$
p^{\prime}=(1-\delta) R+\delta\left(\frac{3}{2} c-\frac{t}{s}\right) .
$$

This equilibrium is well-defined if $R-p^{\prime}>0$ or $R>(3 / 2) c-t / s$. Comparing the prices under both regimes, we have:

$$
p^{*}-p^{\prime}=\frac{\delta}{2 s}(c s-2 t)>0 .
$$

Thus, like in the main text, the equilibrium price is higher in the case of negotiating only with the more efficient providers.

Again, it is straightforward to check that all providers make strictly positive profits:

$$
p^{\prime}-c=(1-\delta) R+\delta\left(\frac{3}{2} c-\frac{t}{s}\right)-c>\frac{3}{2} c-\frac{t}{s}-c=\frac{c s-2 t}{2 s}>0,
$$

since $c s>3 t$. 